\title{
Effects of postnatal overfeeding and fish oil diet on energy expenditure in rats
}

\author{
Yanyan Dai ${ }^{1}$, Nan Zhou ${ }^{1}$, Fan Yang ${ }^{1}$, Shanshan Zhou ${ }^{1}$, Lijun Sha ${ }^{1}$, Jianping Wang ${ }^{2}$ and Xiaonan Li $^{1}$
}

BACKGROUD: Early life nutrition may have a role in the regulation of metabolism in adulthood. The present study aimed to evaluate the effects of postnatal overfeeding and a postweaning fish oil diet on energy expenditure.

METHODS: On postnatal day 3, rat litters were adjusted to a litter size of three (small litters, SLs) or ten (normal litters, NLs). After weaning, SLs were fed the standard diet or a fish oil diet enriched with polyunsaturated fatty acids (SL-FOs) for 10 weeks. The metabolic parameters of rats were monitored using the TSE LabMaster at postnatal week $3\left(\mathrm{~W}_{3}\right)$ and postnatal week $13\left(\mathrm{~W}_{13}\right)$.

RESULTS: At $W_{3}$, the $\mathrm{O}_{2}$ consumption and heat production in SLs were lower than those in NLS, while the respiratory exchange ratio (RER) was higher than NLs. SLs showed obesity, dyslipidemia, and impaired glucose tolerance at $W_{13}$. The postweaning fish oil diet in SLs not only increased $\mathrm{O}_{2}$ consumption, $\mathrm{CO}_{2}$ production, heat production, and reduced the RER but it also reduced weight gain, serum triglycerides, and improved glucose tolerance at $W_{13}$.

CONCLUSION: Postnatal overfeeding can decrease the level of body energy expenditure and induce obesity, but a fish oil diet can increase the energy expenditure and prevent the development of metabolic dysregulation in adults.

E nergy homeostasis is crucial to the maintenance of health. When energy balance is disrupted, energy intake is greater than the output, and the excess energy is stored as triacylglycerols in adipocytes, which eventually induces the development of obesity(1). Obesity is a major risk factor for the development of metabolic diseases such as type 2 diabetes and cardiovascular disease(2), and has become a major health problem worldwide(3). Moreover, $85 \%$ of obese children remain obese in adulthood and also have metabolic dysregulation(4). Therefore, it is crucial to find an effective way to prevent or treat childhood obesity.

The developmental origins of health and disease (DOHaD) hypothesis describe the process by which environmental stimuli, including altered nutrition, during critical periods of development can program alterations in organogenesis, tissue development, and metabolism, predisposing the offspring to metabolic diseases during adulthood(5). Human and animal studies have demonstrated that exposure to overnutrition in early life may increase adiposity and insulin resistance in adulthood(6-8). Moreover, substantial evidence has indicated that dietary fatty acids, physical activity, or lower-calorie diets may change the susceptibility of metabolic diseases(9-11). Fish oil enriched with $\omega 3$ polyunsaturated fatty acids ( $\omega 3$ PUFA) may reduce the development of type 2 diabetes, hyperlipidemia, hypertension, and other metabolic diseases (12-14). In the United States, increasing the amount of dietary $\omega 3$ PUFA may have a role in the treatment of childhood obesity complicated by hyperlipidemia since 2014 (15). Some studies have shown that a significant increase in energy expenditure is accompanied by weight loss in both lean and obese individuals $(16,17)$. However, the effect of $\omega 3$ PUFA on energy expenditure in early nutrition is uncertain.

In this study, early life overfeeding was simulated by adjusting the male Sprague-Dawley rat litter size and intervening with a $\omega 3$ PUFA diet for 10 weeks after weaning. The aim of the present study was to investigate the effects of postnatal overfeeding and $\omega 3$ PUFA on energy expenditure in rats.

\section{METHODS}

\section{Animals}

This study was carried out in accordance with the recommendations for the care and use of laboratory animals of the Department of Experimental Animals of Nanjing Medical University. The study was approved by the Committee on the Ethics of Animal Experiments of Nanjing Medical University (Permit number: 20130102-01). Sprague-Dawley rats (Nanjing, Jiangsu, China) were maintained with a $12 \mathrm{~h}$ light/dark cycle (light cycle, 06:00-18:00; dark cycle, 18:00-06:00) and temperature $\left(22 \pm 2{ }^{\circ} \mathrm{C}\right)$ conditions with free access to tap water and the standard laboratory rodent diet. Except for the intraperitoneal glucose tolerance test, the rats did not undergo invasive operations in this experiment. In addition, the TSE LabMaster (TSE Systems, Bad Homburg, Germany) was used to imitate the normal living environment of rats, and rats were not given analgesics during this study. At the end of the experiment, the rats were anesthetized by an intraperitoneal injection of chloral hydrate after overnight fasting $(12 \mathrm{~h})$. All efforts were made to minimize suffering and to reduce the number of animals used in this study. There were no unintended deaths of rats in this experiment.

\footnotetext{
${ }^{1}$ Department of Child Health Care, Children's Hospital of Nanjing Medical University, Nanjing, China; ${ }^{2}$ Department of Clinical Laboratory, Nanjing Medical University Affiliated Secondary Hospital, Nanjing, China. Correspondence: Xiaonan Li (xiaonan6189@163.com) 


\section{Experimental design}

Female rats were time-mated, and male Sprague-Dawley rat pup litters were randomly distributed among the mothers to adjust to litter sizes of three pups per litter or ten pups per litter to induce early postnatal overfeeding or normal feeding on postnatal day $3\left(\mathrm{P}_{3}\right)$ (18). After weaning, the NL pups were fed with the standard laboratory rodent diet (NL group)(19), and the SL rats were given either the standard laboratory rodent diet (SL group) or a $\omega 3$ PUFA diet (SL-FO group) for 10 weeks. The diets and their nutrient compositions are shown in Table 1. All animals were housed three per cage postweaning. Body weight and food intake were monitored throughout life.

At $\mathrm{W}_{3}$ and $\mathrm{W}_{13}$, rats were monitored in a custom cage with an indirect calorimetry and locomotor activity monitoring system (TSE LabMaster)(20). The calorimetric system of the instrument was an open-circuit system that determined $\mathrm{O}_{2}$ consumption, $\mathrm{CO}_{2}$ production, and respiratory exchange ratio $(\mathrm{RER}=V \mathrm{co} 2 / \mathrm{Vo} 2)$. A photobeam-based activity-monitoring system detected and recorded every ambulatory movement of rats in each cage. To acclimate to the test chambers, rats were placed in the metabolic cages for $72 \mathrm{~h}$, and then were monitored for an additional $24 \mathrm{~h}$. The data from the final $24 \mathrm{~h}$ were used to calculate all parameters for which the results were reported, and the cumulative energy expenditure and locomotor activity during $24 \mathrm{~h}$ were calculated. The rats had free access to food and tap water while they were in the custom cages.

\section{Body Temperature}

At $\mathrm{W}_{3}$ and $\mathrm{W}_{13}$, the body temperature of the rats was assessed by measuring rectal temperature using a rectal thermoprobe (MC-347 Series Precision Thermometers, Omron Temperature, Dalian, China).

\section{Serum and tissue collection}

The rats were anesthetized by an intraperitoneal injection of chloral hydrate $(300 \mathrm{mg} / \mathrm{kg}$ of body weight) after overnight fasting $(12 \mathrm{~h})$. Blood was obtained from the right ventricle and the serum was separated by centrifugation at $2,000 \mathrm{~g}$ for $15 \mathrm{~min}$. The separated serum was stored at $-70^{\circ} \mathrm{C}$ for subsequent determination of biochemical parameters. Epididymal and retroperitoneal white adipose tissue was dissected and weighed, and all tissues were snap-frozen in liquid nitrogen and stored at $-70^{\circ} \mathrm{C}$.

Intraperitoneal glucose tolerance test

The intraperitoneal glucose tolerance test (IPGTT) was performed as

Table 1. Purified diet formula and composition

\begin{tabular}{lcc}
\hline & Soybean oil diet & Fish oil diet \\
\hline Casein & $18.92 \%$ & $18.92 \%$ \\
L-cystine & $0.28 \%$ & $0.28 \%$ \\
Corn starch & $48.34 \%$ & $48.34 \%$ \\
Maltodextrin & $3.32 \%$ & $3.32 \%$ \\
Sucrose & $13.00 \%$ & $13.00 \%$ \\
Cellulose & $4.74 \%$ & $4.74 \%$ \\
Mineral mix & $4.26 \%$ & $4.26 \%$ \\
Vitamin mix & $1.14 \%$ & $1.14 \%$ \\
Soybean oil & $6.00 \%$ & $0.00 \%$ \\
Fish oil & $0.00 \%$ & $6.00 \%$ \\
Total & $100.00 \%$ & $100.00 \%$ \\
Energy (kcal/100 g) & 392.60 & 392.60 \\
$\omega 6 / \omega 3$ ratio & 6.62 & 0.06 \\
\hline
\end{tabular}

previously described. Briefly, rats were fasted overnight at $\mathrm{W}_{13}$. A blood sample was then taken from a tail vein, and $2.0 \mathrm{~g}$ of D-glucose (50\% stock solution in saline) per kilogram of body weight was intraperitoneally injected. Blood was collected from a tail vein at 30-, 60-, and 120-min intervals after the glucose injection, and glucose levels were measured by a glucose meter (Accu-Chek, Roche Diagnostics, Mannheim, Germany).

\section{Biochemical Analysis}

Serum total cholesterol, triglyceride, and high-density lipoprotein were measured using enzymatic colorimetric assays according to the protocols of the commercial clinical diagnosis kits (TCHOD-PAP reagent kit 20090, GPO-PAP reagent kit 20080, BIOSINO BIO, Beijing, China) in an Olympus AU400 analyzer (Olympus Diagnostics, Holliston, USA).

\section{Statistical Methods}

Values were plotted as the mean \pm SD. Significant differences between NLs and SLs of rats at $\mathrm{W}_{3}$ and $\mathrm{W}_{13}$ were analyzed using unpaired $t$-tests. At $\mathrm{W}_{13}$, statistical analyses between groups of rats were performed using one-way analysis of variance (ANOVA) followed by a post hoc Bonferroni test. A $P$-value $<0.05$ was considered to be statistically significant.

\section{RESULTS}

Body Weight, Adipose Tissue Weight, and Food Intake

The body weight of SLs was significantly increased compared with that of NLs at $\mathrm{W}_{3}(P<0.001$, Figure 1$)$, which persisted to $\mathrm{W}_{13}(P<0.01$, Figure 1$)$. In contrast, the body weight of SLFOs was lower than SLs $(P<0.001$, Figure 1) and did not significantly differ from NLs from $\mathrm{W}_{10}$ to $\mathrm{W}_{13}$. The fat pad (retroperitoneal and epididymal) weights of SLs $(0.24 \pm 0.01$ and $0.23 \pm 0.01 \mathrm{~g})$ were higher than NLs $(0.13 \pm 0.01$ and $0.14 \pm 0.01 \mathrm{~g}$, respectively, $P<0.001$, Supplementary Figure $\mathbf{S 1}$ online) at $\mathrm{W}_{3}$, and the difference in fat pad weights in both groups $(9.00 \pm 1.28$ vs. $2.70 \pm 0.27 \mathrm{~g}$ and $8.30 \pm 0.66$ vs. $2.97 \pm 0.18 \mathrm{~g}$, respectively, $P<0.001$, Supplementary Figure S1) still existed at $\mathrm{W}_{13}$. In contrast, the fat pad weight of SL-FOs $(3.17 \pm 0.35$ and $3.80 \pm 0.23 \mathrm{~g})$ was lower than SLs $(9.00 \pm 1.28$ and $8.30 \pm 0.66 \mathrm{~g}$, respectively, $P<0.001$, Supplementary Figure S1) and was not different from NLs.

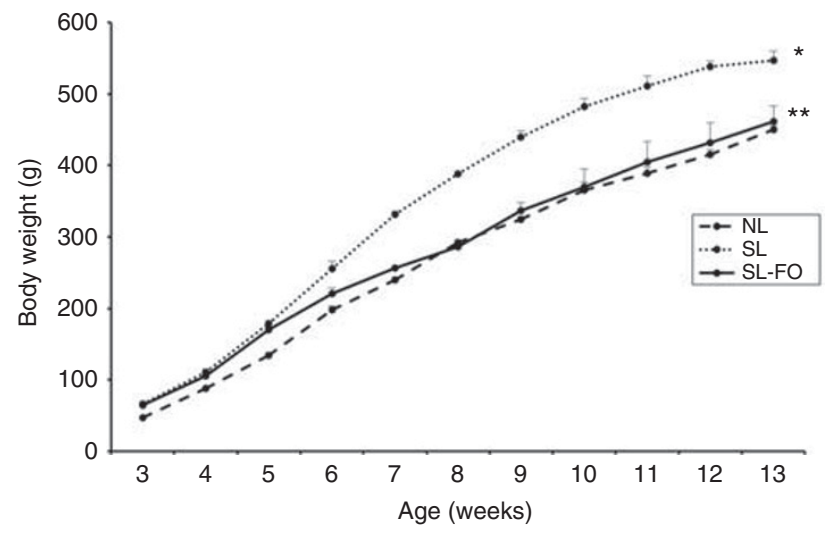

Figure 1. Effect of different diets on body weight from $W_{3}$ to $W_{13}$. The data are expressed as the mean $\pm S D$, and the differences between the groups from $W_{3}$ to $W_{13}$ were analyzed using one-way ANOVA. ${ }^{*} P<0.05$ vs. NL at every time point, ${ }^{* *} P<0.05$ vs. SL from $W_{4}$ to $W_{13}$. The body weight of SL-FO rats was same as that of NL rats from $W_{10}$ to $W_{13}(n=6$ in each group). 


\section{Articles | Dai et al.}

The SLs $(9.60 \pm 0.22 \mathrm{~g})$ exhibited greater food intake compared to NLs $(7.05 \pm 0.20 \mathrm{~g}, P<0.001$, Supplementary Figure S2) at $W_{3}$, and no differences were found in food intake among NLs, SLs, and SL-FOs at $\mathrm{W}_{13}$.

\section{Glucose Homeostasis}

The area under the curve (AUC) for plasma glucose levels over time was calculated following the glucose tolerance test. Compared with NLs (933.00 \pm 30.59$)$, early overfeeding in SLs increased the AUC $(1,195.25 \pm 62.23, P<0.001$, Figure $2 b)$ at $\mathrm{W}_{13}$, and a $\omega 3$ PUFA diet reduced the AUC in SLs $(930.50 \pm 29.99, P<0.001$, Figure $2 \mathbf{b})$ and recovered the plasma glucose to a normal level.

\section{Serum Lipids}

At $\mathrm{W}_{13}$, the total triglyceride level in SLs $(0.77 \pm 0.10 \mathrm{mmol} / \mathrm{l})$ was higher than NLs $(0.40 \pm 0.05 \mathrm{mmol} / \mathrm{l}, \quad P<0.001$, Figure 3a), and was similar between SL-FOs $(0.42 \pm 0.04 \mathrm{mmol} / \mathrm{l})$ and NLs $(0.40 \pm 0.05 \mathrm{mmol} / \mathrm{l}, P=0.81$, Figure 3a). The high-density lipoprotein level in SL-FOs $(0.61 \pm 0.04 \mathrm{mmol} / \mathrm{l})$ was higher than NLs $(0.46 \pm 0.01 \mathrm{mmol} /$ $1, P=0.01$, Figure $3 \mathbf{b}$ ) at $\mathrm{W}_{13}$.

\section{Body Temperature}

The basal rectal temperature was analyzed, showing that the rectal temperature in NLs $\left(36.82 \pm 0.29^{\circ} \mathrm{C}\right)$ at $\mathrm{W}_{3}$ was higher than that in SLs $\left(35.73 \pm 0.34^{\circ} \mathrm{C}, P=0.04\right)$ and persisted to
$\mathrm{W}_{13}\left(35.35 \pm 0.17\right.$ vs. $34.03 \pm 0.13^{\circ} \mathrm{C}$, respectively, $\left.P<0.01\right)$. Furthermore, the rectal temperature in SL-FOs $\left(35.10 \pm 0.24^{\circ}\right.$ C) significantly increased compared to that in SLs $\left(34.03 \pm 0.13^{\circ} \mathrm{C}, P<0.01\right)$ and recovered to a normal level (Table 2).

\section{Energy Expenditure and Ambulatory Activity: $\mathrm{O}_{2}$ Consumption and $\mathrm{CO}_{2}$ Production}

As shown in Figure 4, the $\mathrm{O}_{2}$ consumption of SLs $(3,621.07 \pm 206.81 \mathrm{ml} / \mathrm{h} / \mathrm{kg})$ was lower than NLs $(4,007.25 \pm 286.90 \mathrm{ml} / \mathrm{h} / \mathrm{kg}, \quad P=0.02, \quad$ Figure $\quad 4 a)$ after weaning and persisted to adulthood $(1,397.49 \pm 124.75$ vs. $1,810.79 \pm 78.80 \mathrm{ml} / \mathrm{h} / \mathrm{kg}$, respectively, $P<0.001$, Figure $4 \mathrm{c}$ ). Compared with NLs $(1,653.90 \pm 75.00 \mathrm{ml} / \mathrm{h} / \mathrm{kg})$, the $\mathrm{CO}_{2}$ production of SLs $(1,295.47 \pm 150.61 \mathrm{ml} / \mathrm{h} / \mathrm{kg}, \quad P<0.001$, Figure 4d) was significantly reduced at $\mathrm{W}_{13}$. However, the $\mathrm{O}_{2}$ consumption in SL-FOs $(1,754.00 \pm 188.04 \mathrm{ml} / \mathrm{h} / \mathrm{kg})$ was higher than SLs $(1,397.49 \pm 124.75 \mathrm{ml} / \mathrm{h} / \mathrm{kg}, \quad P<0.001$, Figure 4c), as was $\mathrm{CO}_{2}$ production $(1,579.54 \pm 151.76$ vs. $1,295.46 \pm 150.61 \mathrm{ml} / \mathrm{h} / \mathrm{kg}$, respectively, $P<0.001$, Figure $4 d$ ). The $\mathrm{O}_{2}$ consumption in SL-FOs was more than that in the SLs and was not statistically different from that in the NLs $(1,810.8 \pm 78.80 \mathrm{ml} / \mathrm{h} / \mathrm{kg}, P=0.487)$. The same was true for $\mathrm{CO}_{2}$ production $(1,579.54 \pm 151.76$ vs. $1,653.90 \pm 74.99 \mathrm{ml} / \mathrm{h} /$ $\mathrm{kg}$, respectively, $\mathrm{P}=0.34$ ) in SL-FOs. Moreover, $\mathrm{O}_{2}$ consumption and $\mathrm{CO}_{2}$ production during the day were lower than that at night in NLs at $\mathrm{W}_{13}$ (data not shown).
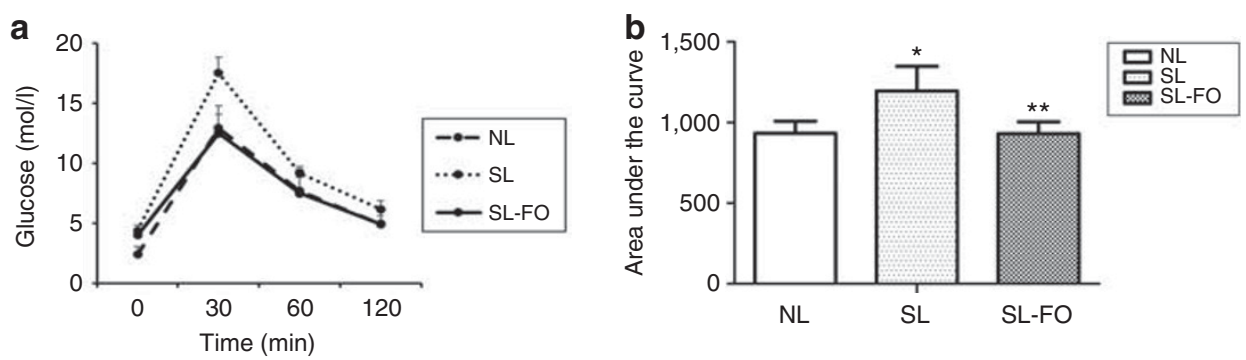

Figure 2. The intraperitoneal glucose tolerance test (a) and areas under the curve of rats $(\mathbf{b})$ at $\mathrm{W}_{13}$. The data are expressed as the mean $\pm \mathrm{SD}$. Differences were analyzed using one-way ANOVA at $\mathrm{W}_{13}{ }^{*} P<0.05$ for SL vs. NL, ${ }^{* *} P<0.05$ for SL-FO vs. SL $(n=6$ in each group).
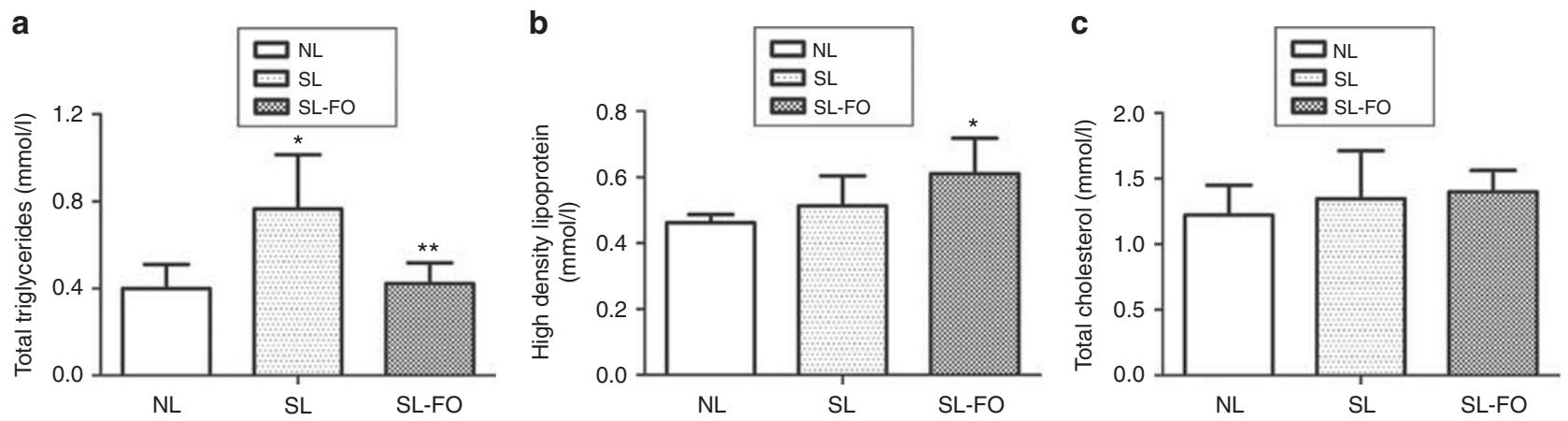

Figure 3. The serum levels of total cholesterol (a), high-density lipoprotein (b), and total triglycerides (c) of rats at $W_{13}$. The data are expressed as the mean \pm SD. Differences were analyzed using one-way ANOVA at $\mathrm{W}_{13} .{ }^{*} P<0.05$ for SL vs. NL, ${ }^{* *} P<0.05$ for SL-FO vs. SL ( $n=6$ in each group). 


\section{Respiratory Exchange Ratio}

Compared with NLs $(0.93 \pm 0.01)$, SL rearing induced a significant increase in RER $(0.96 \pm 0.02, P=0.01$, Figure 5a) at $\mathrm{W}_{3}$. After weaning, there was no difference observed in the RER between NLs and SLs, given the standard diet for 10 weeks. However, the RER of SL-FOs $(0.90 \pm 0.02)$ was lower than that of SLs $(0.92 \pm 0.03, P=0.04$, Figure $5 \mathbf{b})$ and was not statistically different from NLs. Moreover, the RER in NLs and SL-FOs during the day was lower than that at night at $\mathrm{W}_{13}$, but there was no difference in SLs (data not shown).

\section{Heat Production}

Heat production during the day was lower than that at night at $\mathrm{W}_{13}$ in $\mathrm{NL}, \mathrm{SL}$, and SL-FO rats (data not shown). Moreover, the total heat production in SLs $(18.11 \pm 1.04 \mathrm{kcal} / \mathrm{h} / \mathrm{kg})$ was lower than that in NLs

Table 2. Body temperature at $\mathrm{W}_{3}$ and $\mathrm{W}_{13}\left({ }^{\circ} \mathrm{C}\right)$

\begin{tabular}{|c|c|c|c|}
\hline & NL & SL & SL-FO \\
\hline$W_{3}$ & $36.82 \pm 0.71$ & $35.73 \pm 0.84^{*}$ & - \\
\hline$W_{13}$ & $35.35 \pm 0.42$ & $34.03 \pm 0.31^{*}$ & $35.10 \pm 0.58^{* *}$ \\
\hline \multicolumn{4}{|c|}{$\begin{array}{l}\text { FO, fish oil; NL, normal litter; } S L \text {, small litter; } W_{3} \text {, postnatal week } 3 ; W_{13} \text {, postnatal } \\
\text { week } 13 \text {. } \\
\text { Data are the mean } \pm S D, n=6 \text { in each group. } \\
{ }^{*} P<0.05 \text { vs. NL. } \\
{ }^{* * P}<0.05 \text { vs. SL. }\end{array}$} \\
\hline
\end{tabular}
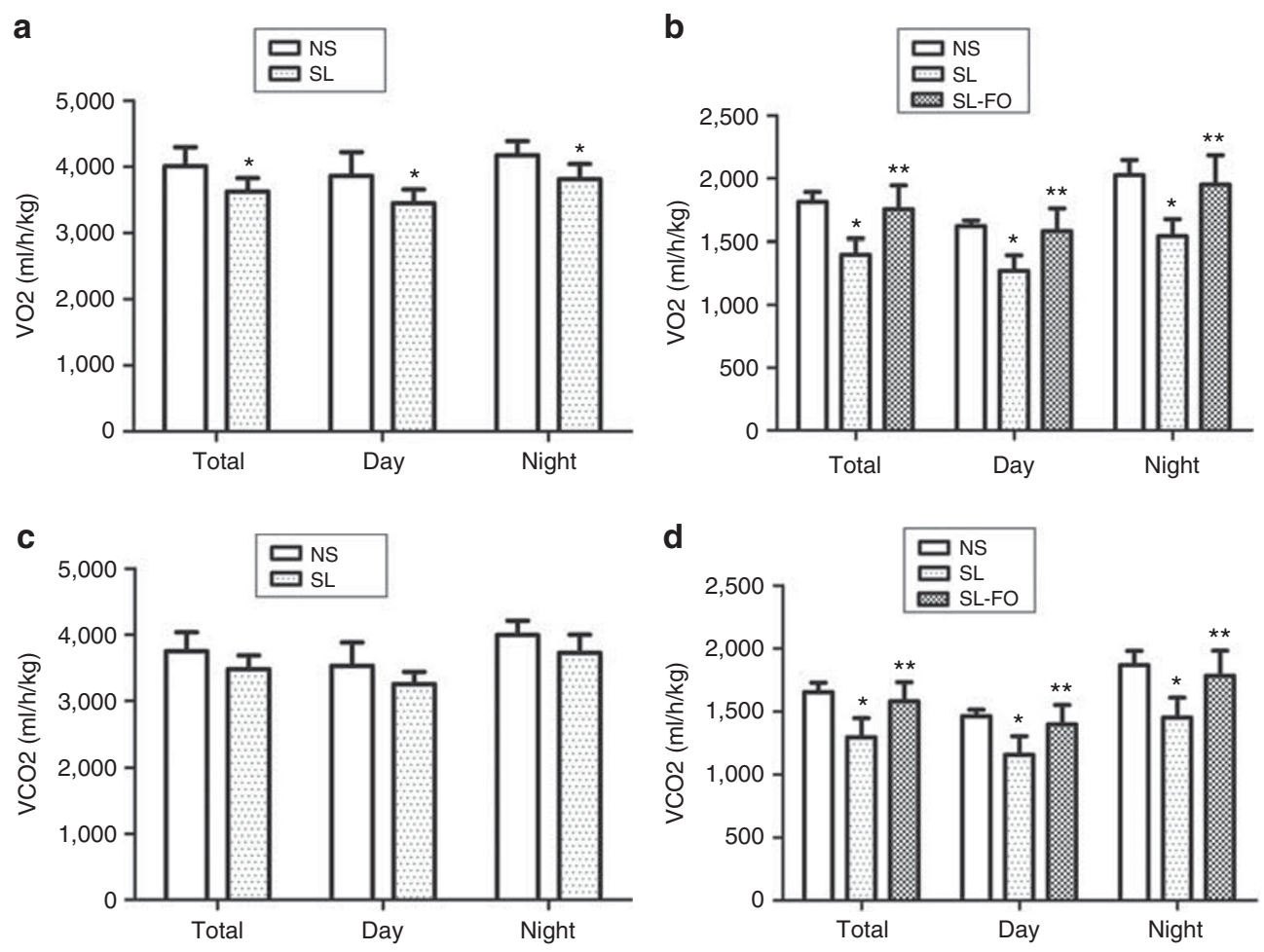

Figure 4. The oxygen consumption and carbon dioxide production of rats at $W_{3}(\mathbf{a}, \mathbf{c})$ and $W_{13}(\mathbf{b}$, d). The data are expressed as the mean $\pm S D$. Differences between groups at $W_{3}$ were analyzed using Student's unpaired $t$-test and using one-way ANOVA at $W_{13} .{ }^{*} P<0.05$ for $S L$ vs. NL, ${ }^{* * *} P<0.05$ for SL-FO vs. SL ( $n=6$ in each group). 


\section{Articles | Dai et al.}

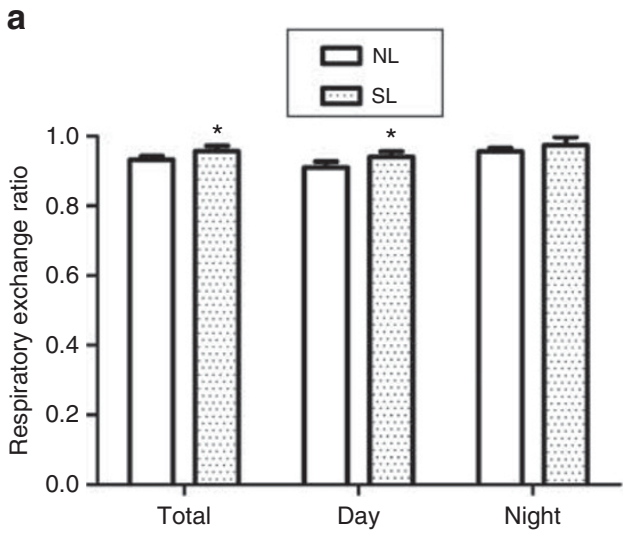

b

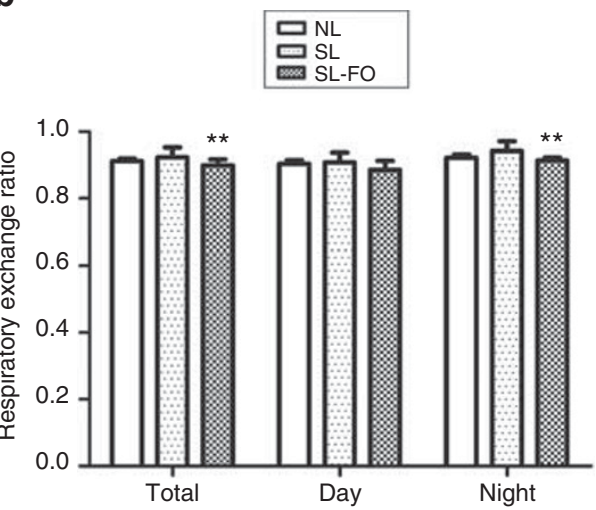

Figure 5. The respiratory exchange ratio of rats at $W_{3}(\mathbf{a})$ and $W_{13}(\mathbf{b})$. The data are expressed as the mean $\pm S D$. Differences between groups at $W_{3}$ were analyzed using Student's unpaired $t$-test and using one-way ANOVA at $\mathrm{W}_{13}{ }^{*} \mathrm{P}<0.05$ for SL vs. NL, ${ }^{* *} P<0.05$ for SL-FO vs. SL ( $n=6$ in each group).
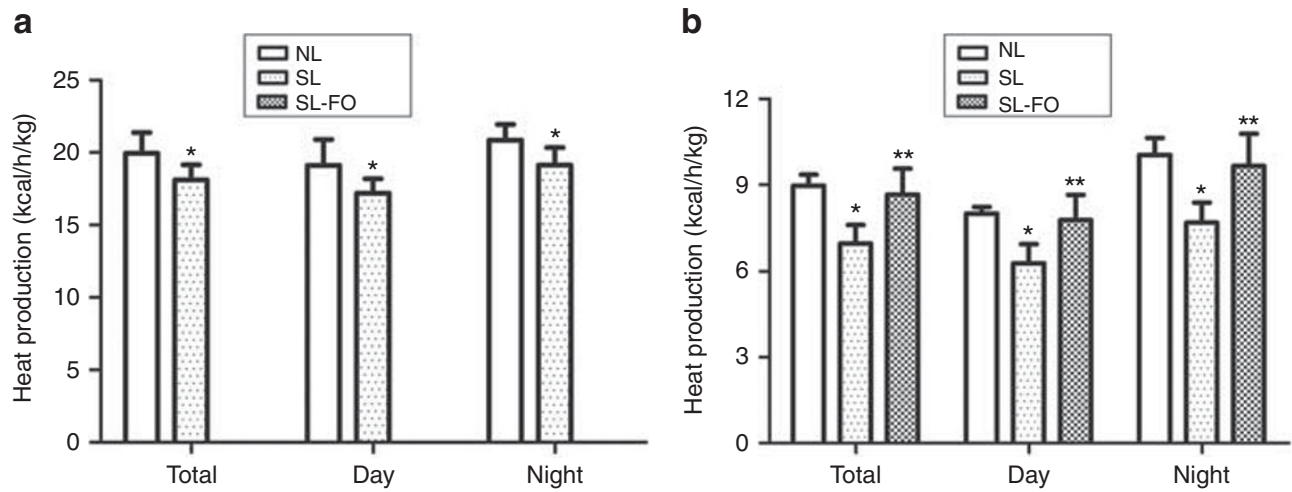

Figure 6. The heat production of rats at $W_{3}(\mathbf{a})$ and $W_{13}(\mathbf{b})$. The data are expressed as the mean $\pm S D$. Differences between groups at $W_{3}$ were analyzed using Student's unpaired $t$-test and at $\mathrm{W}_{13}$ using one-way ANOVA. ${ }^{*} P<0.05$ for SL vs. NL, ${ }^{* *} P<0.05$ for SL-FO vs. SL ( $n=6$ in each group).

may induce a programming effect on energy expenditure, which may contribute to the development of obesity and other metabolic alterations in adult rats. At the same time, a $\omega 3$ PUFA diet reversed the programming effects induced by early postnatal overfeeding on energy expenditure, glucose intolerance, blood lipid levels, and weight gain.

Overnutrition that occurs early in life increases the susceptibility of obesity and the related metabolic diseases (22). Overnutrition during postnatal life can be induced by reducing the litter size, as first demonstrated by Kennedy in 1957(23). It is usually expected that more milk would be available to the individual pups when the litter size is decreased below that of normal delivery, thereby imposing a state of persistent postnatal overfeeding upon the suckling animals. Manipulation of rat litter rearing to induce postnatal overfeeding has been widely used to study the major shortand long-term outcomes of childhood obesity(24). Consistent with these studies, we proved that the body weight and adiposity in SLs were higher than those of NLs, and the difference in body weight and adiposity continued from weaning to adulthood. The regulation of energy balance includes a matching of energy intake with expenditure in order to maintain a stable body weight over an extended period of time(25). The weight gain of SLs may be associated with energy imbalance, but the contribution of energy intake and expenditure to weight gain is still under debate. Given 3 weeks of SL rearing, the food intake of SLs was only more than that of NLs in the first 2 weeks after weaning. There were no differences in food and energy intake from $\mathrm{W}_{5}$ to $\mathrm{W}_{13}$, but SLs still became obese in adulthood. Therefore, we hypothesized that overfeeding early in life could change the development model of energy metabolism, decrease the energy output, and lead to the development of obesity in adulthood.

The reduction in energy expenditure has been a risk factor for weight gain in humans and animals(26-28). In normal individuals, energy is expended mainly by the resting metabolic rate and the thermic effect of food and activity (29). Body energy metabolism is monitored by direct calorimetry and indirect calorimetric measurements(30). Respirometry measures the exchange of respiratory gases with the rat's environment, providing an accurate parameter of heat production (an indicator of energy expenditure) from measurements of $\mathrm{CO}_{2}$ production and $\mathrm{O}_{2}$ consumption(31), and is the main calorimetric method for the indirect measurement of the metabolic rate of rats(32). Oxygen is 
transported to systemic tissues by the cardiovascular system and is used to produce adenosine triphosphate in the mitochondria. Therefore, oxygen consumption can determine the amount of energy that the body aerobically produces(33). Our results indicated that SLs induced a reduction of $\mathrm{O}_{2}$ consumption and maintained it to adulthood, even when a standard diet was consumed. In addition to this, postnatal overfeeding reduced the $\mathrm{CO}_{2}$ production of SLs at $\mathrm{W}_{13}$. The first law of thermodynamics states that energy is only converted from one form into other forms, and is not destroyed or created(1). Animals obtain energy from the oxidation of fatty acids, glucose, and other energy materials at the cell and tissue levels(34). Energy is stored in the form of adenosine triphosphate, and the remaining energy is released in the form of heat(35). The heat production of rats experiencing postnatal overfeeding was significantly lower than that of NLs from $\mathrm{W}_{3}$ to $\mathrm{W}_{13}$, and our data also showed that SLs exhibited a decrease in rectal temperature, indicating that the nutritional state in early life may have had a programming effect on the energy expenditure of SLs in later life.

In addition to food intake and energy expenditure, the composition of the substrate mix used for energy generation also affects energy metabolism $(27,36)$. The RER is derived as an adjustment for the utilization of fuel, and reflects the source of fuel under examined conditions(37). A high RER indicates that carbohydrates are predominantly being used, whereas a low RER suggests lipid oxidation(38,39). In the present study, we found that small litters increased the RER and reduced $\mathrm{O}_{2}$ consumption at weaning, and there was no difference observed in RER between NLs and SLs, given the standard diet after weaning. It has been demonstrated that the milk consumption in SLs is higher than that in NLs, particularly milk triglyceride(24). After weaning, food composition and consumption in SLs showed no differences from those of NLs. Therefore, we may speculate that the different mode of RER at weaning and adulthood of SL rats may be related to nutrient intake. The increased RER in the SL model suggests a reduction in the mobilization of stored lipids, and that the energy source switched to glucose, which reduced lipid oxidation and increased adiposity. In addition, the oxidation of glucose requires less $\mathrm{O}_{2}$ consumption.

Studies have suggested that the composition of fatty acids in a diet may affect the metabolism differentially, thus playing a role in the development or prevention of obesity. High-fat diets may lead to excess energy intake and storage in the adipose tissue(40). In contrast to saturated fatty acids, increasing the amount of PUFAs in the diet plays an important role in hypolipidemic and weight-reducing properties(41). In addition, $\omega 3$ PUFA improves the metabolic profile by upregulation of thermogenic markers in brown adipose tissue(42), promoting beige adipocyte formation and mitochondrial function(43). In this study, postweaning intervention with fish oil rich in $\omega 3$ PUFA decreased body weight gain, improved insulin sensitivity and hyperlipidemia, increased $\mathrm{CO}_{2}$ production, $\mathrm{O}_{2}$ consumption, and heat production, and recovered rectal temperature effectively in SL-FO rats. In addition, the fish oil diet increased fat oxidation and changed fuel utilization by decreasing the RER in SL-FO rats.

Energy intake and physical activity are known to increase the total energy expenditure. In addition, cold exposure in mammals may minimize heat dissipation and increase heat generation(44). However, we found that postnatal overfeeding and 13 PUFA dietary intervention did not affect the physical activities or normal circadian rhythm of rats, and the experimental model or the number of samples could be responsible for this finding. In addition, the TSE system is equipped with heating and cooling equipment to keep the chamber at a constant uniform temperature. Therefore, the effects of temperature, dietary energy, and activity amount on the energy expenditure of the rats were eliminated.

In the present study, we demonstrated that the early postnatal environment could have a programming influence on body weight and energy homeostasis into adulthood. Recent studies have indicated that overnutrition during the immediate postnatal period in rodents leads to permanent effects on the central nervous organization $(45,46)$, circulating concentrations of hormones $(47,48)$, reprogramming of brown adipose tissue-adaptive thermogenesis(49), and obesityrelated gene expression in adipose tissue(50-52) and that these could be the key mechanisms for the occurrence of adverse metabolic outcomes in late life. Therefore, our study is warranted to elucidate the mechanisms of early life programming and dietary intervention on energy expenditure in the future.

In summary, postnatal overfed rats had decreased levels of energy expenditure from postnatal to adulthood and increased body weight and fat weight gain. In contrast, the fish oil diet increased $\mathrm{CO}_{2}$ production, $\mathrm{O}_{2}$ consumption, and heat production and prevented excess adipose accumulation in early life overfed rats. Some studies have raised the possibility that fish oil diets may be a potential therapeutic option for preventing and reducing the development of obesity and other metabolic diseases. These findings could help us to better understand the mechanism of dietary $\omega 3$ PUFA in preventing and reducing the adverse programming outcomes in adults.

\section{SUPPLEMENTARY MATERIAL}

Supplementary material is linked to the online version of the paper at http://www.nature.com/pr

\section{ACKNOWLEDGMENTS}

We thank the Department of Experimental Animals of Nanjing Medical University for their technical assistance.

\section{AUTHOR CONTRIBUTIONS}

Conceived and designed the experiments: Y.D. and X.L. Performed the experiments: Y.D., N.Z., F.Y., S.Z., L.S., and J.W. Analyzed the data: Y.D. and X.L. Wrote the paper: Y.D. and X.L. 


\section{STATEMENT OF FINANCIAL SUPPORT}

This work was financially supported by the Natural Science Foundation of China (81273064), National Program on Key Basic Research Project (2013CB530604), Jiangsu Province Social Development Research (BE2015607), and Key Program of Nanjing Public Health Bureau (ZKX14011).

Disclosure: The authors declare no conflict of interest.

\section{REFERENCES}

1. Assaad H, Yao K, Tekwe CD, et al. Analysis of energy expenditure in dietinduced obese rats. Front Biosci (Landmark Ed) 2014;19:967-85.

2. Park J, Morley TS, Kim M, et al. Obesity and cancer-mechanisms underlying tumour progression and recurrence. Nat Rev Endocrinol 2014;10:455-65.

3. Richard D. Cognitive and autonomic determinants of energy homeostasis in obesity. Nat Rev Endocrinol 2015;11:489-501.

4. Must A, Strauss RS. Risks and consequences of childhood and adolescent obesity. Int J Obes Relat Metab Disord 1999;23 (Suppl 2): S2-11.

5. de Boo HA, Harding JE. The developmental origins of adult disease (Barker) hypothesis. Aust N Z J Obstet Gynaecol 2006;46:4-14.

6. Bernardo AF, Cortez E, Neves FA, et al. Overnutrition during lactation leads to impairment in insulin signaling, up-regulation of GLUT1 and increased mitochondrial carbohydrate oxidation in heart of weaned mice. J Nutr Biochem 2016;29:124-32.

7. Ravelli AC, van Der Meulen JH, Osmond C, et al. Obesity at the age of 50 $\mathrm{y}$ in men and women exposed to famine prenatally. Am J Clin Nutr 1999;70:811-6.

8. Plagemann A, Harder T, Schellong K, et al. Early postnatal life as a critical time window for determination of long-term metabolic health. Best Pract Res Clin Endocrinol Metab 2012;26:641-53.

9. Cooper JA, Watras AC, Adams AK, et al. Effects of dietary fatty acid composition on 24-h energy expenditure and chronic disease risk factors in men. Am J Clin Nutr 2009;89:1350-6.

10. Hollowell RP, Willis LH, Slentz CA, et al. Effects of exercise training amount on physical activity energy expenditure. Med Sci Sports Exerc 2009;41:1640-4.

11. Westbrook R, Bonkowski MS, Arum O, et al. Metabolic alterations due to caloric restriction and every other day feeding in normal and growth hormone receptor knockout mice. J Gerontol A Biol Sci Med Sci 2014;69: 25-33.

12. Wang $\mathrm{X}$, Chan CB. $\mathrm{n}-3$ polyunsaturated fatty acids and insulin secretion. J Endocrinol 2015;224:R97-106.

13. Griffin BA. How relevant is the ratio of dietary $n-6$ to $n-3$ polyunsaturated fatty acids to cardiovascular disease risk? Evidence from the OPTILIP study. Curr Opin Lipidol 2008;19:57-62.

14. Stanley JC, Elsom RL, Calder PC, et al. UK Food Standards Agency Workshop Report: the effects of the dietary n-6:n-3 fatty acid ratio on cardiovascular health. Br J Nutr 2007;98:1305-10.

15. Estrada E, Eneli I, Hampl S, et al. Children's Hospital Association consensus statements for comorbidities of childhood obesity. Child Obes 2014;10:304-17.

16. Martin CK, Heilbronn LK, de Jonge L, et al. Effect of calorie restriction on resting metabolic rate and spontaneous physical activity. Obesity 2007;15: 2964-73.

17. Johannsen DL, Knuth ND, Huizenga R, et al. Metabolic slowing with massive weight loss despite preservation of fat-free mass. J Clin Endocrinol Metab 2012;97:2489-96.

18. Rodrigues AL, de Moura EG, Passos MC, et al. Postnatal early overnutrition changes the leptin signalling pathway in the hypothalamic-pituitary-thyroid axis of young and adult rats. J Physiol 2009;587:2647-61.

19. Hou M, Ji C, Wang J, et al. The effects of dietary fatty acid composition in the post-sucking period on metabolic alterations in adulthood: can omega3 polyunsaturated fatty acids prevent adverse programming outcomes? J Endocrinol 2012;215:119-27.
20. Czyzyk TA, Nogueiras R, Lockwood JF, et al. kappa-Opioid receptors control the metabolic response to a high-energy diet in mice. FASEB J 2010;24:1151-9.

21. Cottrell EC, Ozanne SE. Developmental programming of energy balance and the metabolic syndrome. Proc Nutr Soc 2007;66:198-206.

22. Morrison JA, Friedman LA, Wang P, et al. Metabolic syndrome in childhood predicts adult metabolic syndrome and type 2 diabetes mellitus 25 to 30 years later. J Pediatr 2008;152:201-6.

23. Kennedy GC. The development with age of hypothalamic restraint upon the appetite of the rat. J Endocrinol 1957;16:9-17.

24. Habbout A, Li N, Rochette L, et al. Postnatal overfeeding in rodents by litter size reduction induces major short- and long- term pathophysiological consequences. J Nutr 2013;143:553-62.

25. Rothwell NJ, Stock MJ. Regulation of energy balance. Annual Rev Nutr 1981;1:235-56.

26. Blundell JE, Cooling J. Routes to obesity: phenotypes, food choices and activity. Br J Nutr 2000;83:S33-8.

27. Schrauwen P, Westerterp KR. The role of high-fat diets and physical activity in the regulation of body weight. Br J Nutr 2000;84:417-27.

28. Pagliassotti MJ, Gayles EC, Hill JO. Fat and energy balance. Ann N Y Acad Sci 1997;827:431-48.

29. Manini TM. Energy expenditure and aging. Ageing Res Rev 2010;9:1-11.

30. Mercer SW, Trayhurn P. Effect of high fat diets on energy balance and thermogenesis in brown adipose tissue of lean and genetically obese ob/ ob mice. J Nutr 1987;117:2147-53.

31. Arch JR, Hislop D, Wang SJ, et al. Some mathematical and technical issues in the measurement and interpretation of open-circuit indirect calorimetry in small animals. Int J Obes 2006;30:1322-31.

32. Burnett CM, Grobe JL. Dietary effects on resting metabolic rate in C57BL/6 mice are differentially detected by indirect $(\mathrm{O} 2 / \mathrm{CO} 2$ respirometry) and direct calorimetry. Mol Metab 2014;3:460-4.

33. Hanaoka Y, Yasuda $\mathrm{O}$, Soejima $\mathrm{H}$, et al. Tissue inhibitor of metalloproteinase-3 knockout mice exhibit enhanced energy expenditure through thermogenesis. PLoS ONE 2014;9:e94930.

34. Dai Z, Wu Z, Yang Y, et al. Nitric oxide and energy metabolism in mammals. BioFactors 2013;39:383-91.

35. Wu G. Functional amino acids in nutrition and health. Amino Acids 2013;45:407-11.

36. Flatt JP. Dietary fat, carbohydrate balance, and weight maintenance: effects of exercise. Am J Clin Nutr 1987;45:296-306.

37. Xia T, Zhang Q, Xiao Y, et al. CREB/TRH pathway in the central nervous system regulates energy expenditure in response to deprivation of an essential amino acid. Int J Obes 2015;39:105-13.

38. Ramos-Jiménez A, Hernández-Torres RP, Torres-Durán PV, et al. The respiratory exchange ratio is associated with fitness indicators in Trained and Untrained men: a possible application for people with reduced exercise tolerance. Clin Med Circ Respirat Pulm Med 2008;2:1-9.

39. Pendergast DR, Leddy JJ, Venkatraman JT. A perspective on fat intake in athletes. J Am Coll Nutr 2000;19:345-50.

40. Kien CL, Bunn JY, Ugrasbul F. Increasing dietary palmitic acid decreases fat oxidation and daily energy expenditure. Am J Clin Nutr 2005;82: $320-6$.

41. Calder PC. $n-3$ polyunsaturated fatty acids, inflammation, and inflammatory diseases. Am J Clin Nutr 2006;83:1505s-19s.

42. Bargut TC, Silva-e-Silva AC, Souza-Mello V, et al. Mice fed fish oil diet and upregulation of brown adipose tissue thermogenic markers. Eur J Nutr 2016;55:159-69.

43. Fleckenstein-Elsen M, Dinnies D, Jelenik T, et al. Eicosapentaenoic acid and arachidonic acid differentially regulate adipogenesis, acquisition of a brite phenotype and mitochondrial function in primary human adipocytes. Mol Nutr Food Res 2016;60:2065-75.

44. Ravussin Y, Xiao C, Gavrilova O, et al. Effect of intermittent cold exposure on brown fat activation, obesity, and energy homeostasis in mice. PLoS ONE 2014;9:e85876.

45. Gali Ramamoorthy T, Begum G, Harno E, et al. Developmental programming of hypothalamic neuronal circuits: impact on energy balance control. Front Neurosci 2015;9:126. 
46. Plagemann A, Harder T, Rake A, et al. Perinatal elevation of hypothalamic insulin, acquired malformation of hypothalamic galaninergic neurons and syndrome $\mathrm{x}$-like alterations in adulthood of neonatally overfed rat. Brain Res 1999;836:146-55.

47. Collden G, Balland E, Parkash J, et al. Neonatal overnutrition causes early alterations in the central response to peripheral ghrelin. Mol Metab 2014;24:15-24.

48. Boullu-Ciocca S, Tassistro V, Dutour A, et al. Pioglitazone in adult rats reverses immediate postnatal overfeeding-induced metabolic, hormonal, and inflammatory alterations. Endocrine 2015;50:608-19.
49. Xiao XQ, Williams SM, Grayson BE, et al. Excess weight gain during the early postnatal period is associated with permanent reprogramming of brown adipose tissue adaptive thermogenesis. Endocrinology 2007;148:4150-9.

50. Achard V, Sanchez C, Tassistro V, et al. Immediate postnatal overfeeding in rats programs aortic wall structure alterations and metalloproteinases dysregulation in adulthood. Am J Hypertens 2016;29:719-26.

51. Palou A, Picó C, Bonet ML. Nutritional potential of metabolic remodelling of white adipose tissue. Curr Opin Clin Nutr Metab Care 2013;16:650-6.

52. Hou M, Liu Y, Zhu L, et al. Neonatal overfeeding induced by small litter rearing causes altered glucocorticoid metabolism in rats. PLoS ONE 2011;6:e25726. 